\title{
Mast Cells, T regulatory Cells, Myeloid-derived Suppresor Cells and their Role in Prognosis of Different Tumors
}

\author{
Oner Ozdemir* \\ Department of Pediatrics, Research and Training Hospital of Sakarya University, \\ Turkey
}

*Corresponding author: Oner Ozdemir, MD Division of Allergy and Immunology Department of Pediatrics Faculty of Medicine, Sakarya University Research and Training Hospital of Sakarya University, Adnan Menderes Cad., Saglik Sok. No: 195 Adapazarı, Sakarya, Turkey, Tel: 90-(264) -444 54 00; Fax: 90-(264) -275 91 92; Email: ozdemir_oner@hotmail.com

\section{Abstract}

Recent literature data indicate interesting, complex relationships among mast cell, T regulatory cells and myeloid-derived suppressor cells in tumor stroma for immune evasion and tumor prognosis. The negative impact of peritumoral mast cells on the prognosis of various tumors such as in hepatocellular carcinoma has been reported. However, the inhibitory or proliferative effect of mast cells on tumor growth depends on perplexing interactions among mast cell, tumor type, and other immune cells in the tumor microenvironment. The theory defining mast cells and Treg cell's functional interaction has been proposed by several recent reports as well. Mast cells firstly facilitate the accumulation of myeloid-derived suppresor cells into tumor stroma, and these cells could release IL-17 which draws Treg cells and further magnify immunosuppressive effect. Tumor proliferation and immune evasion takes place by the inequity between immune effector cell function and cells with suppressor / regulatory function in tumor stromas.

Keywords: Mast cells; T regulatory cells; Myeloid-derived suppresor cells; Immune evasion; Tumor

\section{Introduction}

Recent literature data describe interesting, complex relationships among mast cell (MC), T regulatory (Treg) cells and myeloid-derived suppressor cell (MDSC) in tumor stroma for immune evasion and the prognosis of tumor. Our interest in these studies is that we have observed in vitro human MC-mediated cytotoxicity against different human tumor (such as leukemia and lymphoma) cells, in contradiction of these mounting literature data $[1,2]$. Here, we briefly comment on quite controversial MC- Treg- MDSC relationship in tumor tissue and on tumor inhibitory role of MCs under the light of recent literature.

\section{Mast Cells in Tumor Tissues}

Mast cells are a bone marrow-derived, long-standing, diverse population functioning as either positive or negative regulators of human innate immune system. Mast cells are one of the most prolific chemical factories in the body and affect other inflammatory and immune cells by means of both soluble mediators and cell-tocell interactions. The negative impact of peritumoral MCs on the prognosis of various tumors such as in lung, kidney 


\section{Cell \& Cellular Life Sciences Journal}

and hepatocellular carcinoma has been reported [3]. These reports also suggest a positive correlation between tumor progression, invasiveness and higher MC density (MCD). Nevertheless, increased MCD has not always been reported to be related with poor prognosis of tumor in human and mice; it can also indicate a good prognosis. For instance: a study showed that peritumoral MC infiltration predicts better survival in advanced ovarian cancer [4]. In another study, MC infiltration was supposed to hamper the progression / dissemination of the gastric carcinoma [5]. In a recent study, stromal MC accumulation in invasive breast cancer patients was found to be an independent good prognostic marker. In addition, high MCD is known to predict a better prognosis in different tumors [6] e.g. human prostate, colo-rectal and bladder cancers. Mice studies have also demonstrated that MCdeficient mice had an augmented tumor incidence when compared with control. The higher incidence reversed to the control level when MC deficiency had been surmounted [7]. A mice study in a genetic model of earlystage intestinal tumorigenesis has indicated a defensive, anti-tumor role of MCs [8]. These paradoxical reports indicate that there seems to be other factors, e.g. variations in biopsy timing, tumor types / stages, and methodology, determining relationship between MCD and tumor progression. We also consider that the inhibitory or proliferative effect of MCs on tumor progression depends on various interactions among MC, tumor type and their relationship with other immune cells in tumor microenvironment. Furthermore, only by demonstrating high MCD in/around a tumor tissue, to make a correlation with poor/good prognosis from samples seems far behind to clarify their genuine role $[1,2,6]$.

MCs are known to be prominent in the tumor stroma and are considered to provide a host response of the innate immunity against tumor $[1,2,4,7,8]$. Nonetheless, some researchers advocate tumor promoting role of MCs attributed to their immunosuppressive function in chronic inflammation and tumor tissues $[3,6]$. They are supposed to be multifunctional and they can act as effector elements of human innate immune system. MCs have been also proven to act as critical upstream regulators of numerous inflammatory cells. In fact, infiltration of MCs may be a fraction of widespread inflammatory or host defense reaction as defined in some tumors, i.e. gastric carcinoma, resembling physiological processes such as in scars and ovulation [5]. Now, the question comes in mind: May the high MCD be a result or a reason of tumor growth or chronic inflammation?
Some authors considered that MC infiltration was related to poor prognosis without taking into account the anti-tumor effects of MCs. On the contrary; growing data suggest that MCs could inhibit tumor progression similar to ours in vitro experiments [1,2]. We have shown in vitro human MC cytotoxicity against various human tumor cells indicating MC's anti-tumor effect $[1,2,6]$. Moreover, tumor cell phagocytosis by stromal MCs in thyroid cancer [9] and in vivo apoptosis of rat hepatoma cells by MCs were also observed [10]. Several other factors, such as antitumor mediators (chymase, TNF- $\alpha$ ) released from MCs and the relationship of MCs with other cell types should be considered as well. For instance, mast cell TNF- $\alpha$ was demonstrated to cause apoptosis in target tumor cells [11]. Lately, cultured MCs have also been proven to contain granzyme B and Fas Ligand which are elements of cell-mediated cytotoxicity of immune effector cells [12]. Chymase was shown to cause apoptosis in neonatal rat cardiomyocytes and human vascular smooth muscle cells [11]. In addition, a number of other mediators from fibroblasts and other cells besides MCs in tumor stroma could potentially affect tumor progression through various mechanisms such as cyclooxygenase metabolites and heparanases [13-16]. The net effects of MCs on tumor growth through immune evasion, therefore, are expected to be the consequence of numerous interactions between the assorted elements of MC granules and nearby stromal cells e.g. vascular endothelium and fibroblasts. With an improvement in the techniques of evaluating MCs, allied inflammatory cells and their signaling pathways, the immune modulatory function of the MCs will be further elucidated. Known the series of mediators potentially secreted by MCs, it is impossible to foresee how MCs will skew a T-cell response without careful contemplation of how the MC is activated and the micro environmental milieu in sight [15]. Whether MCs are precisely capable of suppressing T-cell responses via any one of these pathways in a tumor stroma, however, still remains unanswered [16].

\section{T Regulatory (Treg) Cells' Role in Tumor Stroma}

Treg cells, especially Foxp3+-Tregs, are demonstrated to be available in quite a few kinds of cancers including lung, breast, pancreatic, ovarian, and melanoma. Treg cells can suppress the actions of many types of immune system cells such as CD4+- $\mathrm{T}$ cells, CD8+- $\mathrm{T}$ cells, dendritic cells, B cells, macrophages, osteoblasts, natural killer (NK) cells and NK-T cells, which are significant protector cells of the body to avert cancer genesis and progression [17]. 


\section{Cell \& Cellular Life Sciences Journal}

Recent research has showed that immune suppression caused by Tregs is a main facilitator of immune evasion, but the origins of these cells and their involvement to human tumor growth still remain vague. Other than Tregs, antigen presenting cell (APC)s are also recently demonstrated to be existing as the key players in immune suppression and are accepted as significant contributors to the tolerogenic tumor stroma $[18,19]$. While IL-9 secretion by the Tregs was thought to be vital for their effects on MCs, the accurate immunosuppressive substances released by MCs have not yet been identified. IL-9 has been accepted as a essential factor in the regulation of Treg cells and $\mathrm{MC}$ enrollment and action [17]. On one hand Tregs are thought to play a major role in tumor growth by suppressing protective anti-tumor immune responses, on the other hand a series of new observations imply that Tregs are protective in tumor by virtue of their ability to control tumor-associated inflammation $[17,20,21]$. Interestingly, a recent prostate cancer study revealed that supplementation with syngeneic Treg cells gathered from wild-type mice decreased the IL-6 / IL-9 levels and risk of prostate cancer [22]. Consistently, Treg depletion in 2-month-old animals augmented IL- 6 expression inside prostate and increased the high-grade prostatic cancer incidence and micro invasive carcinoma. Tregs decreased inflammationrelated carcinogenic processes and contributed to immune and epithelial homeostasis [22].

The theory that MCs and Tregs functionally cooperate has been proposed by several recent reports. It has been observed that MCs are infiltrated in the tumor stroma via the stem cell factor (SCF) / c-kit signaling pathway. They gather MDSCs to tumor stroma, and MDSCs could secrete IL-17 which draws Treg cells and intensify immunosuppressive effect more. MCs function as intermediaries in Treg-induced tolerance but can also adapt or reverse Treg-suppressive properties [17,19,21]. Tregs are supposed to facilitate tumor growth by curbing defensive anti-tumor responses. To diminish or to functionally inhibit Treg cells is considered to be one of the potential strategies in the cancer treatment $[3,17]$. Recent clinical and laboratory studies demonstrated that Tregs are a favorable factor against cancer. An insight of the new aspects regarding Treg subsets in tumor and current advances in the recognition of Treg-specific markers, besides the possible strategies to aim Tregs will help to enhance anti-tumor immunity [20, 21, 23, 24]. A recent study has shown that Tregs have both promoting and inhibiting effects on tumors reliant on the stage of tumor growth. By using $0.5 \mathrm{mg}$ cyclophosphamide,
Tregs were constantly and exclusively depleted in a murine liver cancer model. Under such conditions, it was found that tumor growth was reduced at early stages, but promoted later on. Analysis of the tumor stroma revealed that long-term Treg diminution by cyclophosphamide induced Gr-1 (+) / CD11b (+) MDSCs. Ablation of MDSCs by anti-Gr-1 Ab prevented Treg depletion-induced support of tumor development. In this study, Tregs were thought to play different roles at different stages of tumor development: inhibiting at early stage and promoting late tumor progression [25].

\section{Myeloid-Derived Suppresor Cells of Tumor Microenvironment}

Myeloid-derived suppresor cells are originated from bone marrow under the effects of tumor cell- derived substances. In mice MDSC are defined by Gr-1 / CD11b expression, but in human there are no specific markers for this population [26]. MDSC show negative effect on the function of T-cells by arginine depletion and nitric oxide production. Some laboratory experiments in reversing the negative effect of MDSC on immune system are provided, mainly through the applying monoclonal antibodies against MDSC or drugs blocking their function [17]. The clarification of MDSC characteristics can let us formulate new immunotherapy approaches in the future. As mentioned above, MCs induce the accumulation of MDSCs into tumor microenvironment and increase the production of IL-17 by MDSCs; MDSCs-derived IL-17 indirectly draws Tregs, increase their suppressor function, and induce the IL-9 release by Tregs; in turn, IL9 fortify the survival and protumor effect of MCs in tumor stroma [21,26,27]. Consequently, tumor development is caused by the imbalance between immune cells with effector function and cells with suppressor/regulatory functions in tumor stroma [27]. Finally, many studies proved no significant correlation between MC/ Treg/ MDSC and the stage/prognosis of the tumor. And making a correlation between MC/ Treg/ MDSC density and tumor invasiveness is insufficient to evaluate their net effect in tumor development. The evidence/proofs are not enough to define MC/ Treg/ MDSC density and their relations as promising predictors for tumor growth or prognosis in tumor stroma.

\section{Conclusion}

Due to perplexing findings, it is impossible to state that solely Tregs contribute to immune suppression induced by MCs and MDSCs. Thus, could be the increase of MC/ 


\section{Cell \& Cellular Life Sciences Journal}

Treg/ MDSC densityan early event at the onset of tumor development or more likely a response of the immune system during tumor progression? All of the research so far implies that elucidating the roles of MC/ Treg/ MDSC in different human tumors requires more complex studies beyond those assessing merely the density of these cells in the tumor microenvironment. In the age of targeted therapy, studies investigating the MC/ Treg/ MDSC density's role in tumor stroma can have direct clinical consequences and should be further clarified via the use of histopathological and complex biological models. Thus, we think that reversing the infiltration of MC/ Treg/ MDSC in peritumoral liver tissues, as suggested by some authors, may not be an effective strategy to improve current anti-cancer regimens and prolong survival.

\section{References}

1. Ozdemir O (2011) Flow cytometric mast cellmediated cytotoxicity assay: a three-color flow cytometric approach using monoclonal antibody staining with annexin $\mathrm{V} /$ propidium iodide co-labeling to assess human mast cell-mediated cytotoxicity by fluorosphere-adjusted counts. J Immunol Methods 365(1-2): 166-173.

2. Ozdemir O (2007) Evaluation of human mast cellmediated cytotoxicity by DIOC18 target cell labeling in flow cytometry. J Immunol Methods 319(1-2): 98103.

3. Ju MJ, Qiu SJ, Gao Q, Fan J, Cai MY, et al. (2009) Combination of peritumoral mast cells and $\mathrm{T}$ regulatory cells predicts prognosis of hepatocellular carcinoma. Cancer Sci 100(7): 1267-1274.

4. Chan JK, Magistris A, Loizzi V, Lin F, Rutgers J, et al. (2005) Mast cell density, angiogenesis, blood clotting, and prognosis in women with advanced ovarian cancer. Gynecol Oncol 99(1): 20-25.

5. Jiang YA, Zhang YY, Luo HS (2002) Mast cell density and the context of clinicopathological parameters and expression of p185, estrogen receptor, and proliferating cell nuclear antigen in gastric carcinoma. World J Gastroenterol 8(6): 1005-1008.

6. Ozdemir O (2006) Mast cell density, neoplastic angiogenesis and their prognostic importance. Dig Liver Dis 38(5): 356

7. Ponvert C, Galoppin L, Canu P (1994) Modifications in tissue histamine levels in mast celldeficient mice $(\mathrm{W} / \mathrm{Wv})$ and in their littermates $(\mathrm{Wv} /+, \mathrm{W} /+$ and $+/+)$ grafted with a methylcholanthrene-induced fibrosarcoma: correlation with tumour rejection. Cancer Lett 85(1): 125-131.

8. Sinnamon MJ, Carter KJ, Sims LP, Lafleur B, Fingleton B, et al. (2008) A protective role of mast cells in intestinal tumorigenesis. Carcinogenesis 29(4): 880886.

9. Fiumara A, Belfiore A, Russo G, Salomone E, Santonocito GM, et al. (1997) In situ evidence of neoplastic cell phagocytosis by macrophages in papillary thyroid cancer. J Clin Endocrinol Metab 82(5): 1615-1620.

10. Nishikawa M, Sato EF, Kuroki T, Utsumi K, Inoue M. (1998) Macrophage-derived nitric oxide induces apoptosis of rat hepatoma cells in vivo. Hepatology 28(6): 1474-1480.

11. Heikkila HM, Latti S, Leskinen MJ, Hakala JK, Kovanen PT, et al. (2008) Activated mast cells induce endothelial cell apoptosis by a combined action of chymase and tumor necrosis factor-alpha. Arterioscler Thromb Vasc Biol 28(2): 309-314.

12. Zorn CN, Pardo J, Martin P, Kuhny M, Simon MM, et al. (2013) Secretory lysosomes of mouse mast cells store and exocytose active caspase- 3 in a strictly granzyme B dependent manner. Eur J Immunol 43(12): 32093218

13. Marichal T, Tsai M, Galli SJ. (2013) Mast cells: potential positive and negative roles in tumor biology. Cancer Immunol Res 1(5): 269-279.

14. Ferrandina G, Lauriola L, Zannoni GF, Distefano MG, Legge F, et al. (2002) Expression of cyclooxygenase-2 (COX-2) in tumour and stroma compartments in cervical cancer: clinical implications. Br J Cancer 87(10): 1145-1152.

15. Wasiuk A, De Vries VC, Hartmann K, Roers A, Noelle RJ (2009) Mast cells as regulators of adaptive immunity to tumour. Clin Exp Immunology 155 (2): 140-146.

16. Ozdemir 0 (2006) Mast cell density, angiogenesis, and their significance in tumor development. Gynecol 


\section{Cell \& Cellular Life Sciences Journal}

Oncol 100(3): 628-630.

17. Feng LL, Gao JM, Li PP, Wang $X$ (2011) IL-9 contributes to immunosuppression mediated by regulatory $\mathrm{T}$ cells and mast cells in B-cell nonhodgkin's lymphoma. J Clin Immunol 31(6): 10841094.

18. Munn DH, Mellor AL (2006) The tumor-draining lymph node as an immune-privileged site. Immunol Rev 213: 146-158.

19. Khazaie K, Blatner NR, Khan MW, Gounari F, Gounaris E, et al. (2011) The significant role of mast cells in cancer. Cancer Metastasis Rev 30(1): 45-60.

20. Elkord E, Alcantar-Orozco EM, Dovedi SJ, Tran DQ, Hawkins RE, et al. (2010) T regulatory cells in cancer: recent advances and therapeutic potential. Expert Opin Biol Ther 10(11): 1573-1586.

21. Yang Z, Zhang B, Li D, Lv M, Huang C, et al. (2010) Mast cells mobilize myeloid-derived suppressor cells and Treg cells in tumor microenvironment via IL-17 pathway in murine hepatocarcinoma model. PLoS One 5(1): e8922.

22. Poutahidis T, Rao VP, Olipitz W, Jackson EA, Levkovich T, et al. (2009) CD4+ lymphocytes modulate prostate cancer progression in mice. Int J Cancer 125(4): 868-878.
23. Zhang HH, Mei MH, Fei R, Liao WJ, Wang XY, et al. (2010) Regulatory $T$ cell depletion enhances tumor specific CD8 T-cell responses, elicited by tumor antigen NY-ESO-1b in hepatocellular carcinoma patients, in vitro. Int J Oncol 36(4): 841-848.

24. Cany J, Tran L, Gauttier V, Judor JP, Vassaux G, et al. (2011) Immunotherapy of hepatocellular carcinoma: is there a place for regulatory T-lymphocyte depletion? Immunotherapy 3(4): 32-34.

25. Zhang B, Jia H, Liu J, Yang Z, Jiang T, et al. (2010) Depletion of regulatory $\mathrm{T}$ cells facilitates growth of established tumors: a mechanism involving the regulation of myeloid-derived suppressor cells by lipoxin A4. J Immunol 185(12): 7199-7206.

26. Luczynski W, Krawczuk-Rybak M, Stasiak-Barmuta A. (2008) [Myeloid-derived suppressor cells - the new mechanism of immunosuppression in cancer]. Postepy Hig Med Dosw (Online) 62: 18-22.

27. Rosin FC, Pedregosa JF, de Almeida JS, Bueno V. (2011) Identification of myeloid-derived suppressor cells and $\mathrm{T}$ regulatory cells in lung microenvironment after Urethane-induced lung tumor. Int Immunopharmacol 11(7): 873-878. 